\title{
Perfil antropométrico dos idosos residentes em Campina Grande-PB
}

\author{
Anthropometric profile of the elderly residents in Campina Grande-PB, Brazil
}

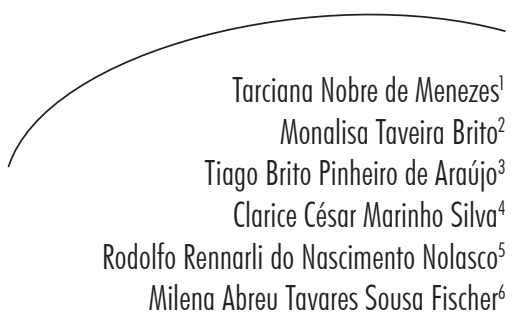

Resumo

O objetivo deste estudo foi descrever o perfil antropométrico dos idosos residentes em Campina Grande - PB. Este estudo é populacional, transversal e domiciliar. Os dados são apresentados sob a forma de média, desvio-padrão e percentil (P5, P10, P25, P50, P75, P90 e P95) do peso, estatura, índice de massa corporal (IMC), perímetro do braço (PB), dobra cutânea tricipital (DCT), circunferência muscular do braço (CMB), perímetro da panturrilha (PP) e perímetro da cintura (PC). Foi realizado o teste $t$ Student para verificar a diferença dos valores médios das variáveis entre os sexos. Foi realizada a análise de variância (ANOVA) para verificar o efeito da idade nas variáveis. Foram avaliados 806 idosos (60 anos ou mais). Os valores médios de todas as variáveis apresentaram diferença estatisticamente significativa entre os sexos. As médias das variáveis peso, estatura, CMB, PP e PC foram significativamente maiores entre os homens, enquanto que as médias de IMC, PB e DCT foram significativamente maiores entre as mulheres. Todas as variáveis apresentaram tendência de declínio entre os grupos etários mais avançados. Os resultados mostram que o padrão antropométrico dos idosos deste estudo segue a tendência de outros estudos, no entanto, os valores diferem. Dessa forma, sugere-se que estes possam ser usados para auxiliar na avaliação antropométrica de idosos.

\footnotetext{
Departamento de Fisioterapia. Programa de Pós-graduação em Saúde Pública. Universidade Estadual da Paraíba. Campina Grande, PB, Brasil.

2 Programa de Pós-graduação em Produtos Naturais e Sintéticos Bioativos. Universidade Federal da Paraíba. João Pessoa, PB, Brasil.

3 Curso de Fisioterapia. Centro de Ciências Biológicas e da Saúde. Universidade Estadual da Paraíba. Campina Grande, PB, Brasil.

4 Departamento de Segurança do Trabalho. Instituto Federal de Pernambuco. Belo Jardim, PE, Brasil.

5 Curso de Enfermagem. Centro de Ciências Biológicas e da Saúde. Universidade Estadual da Paraíba. Campina Grande, PB, Brasil.

6 Instituto de Geriatria e Gerontologia. Pontifícia Universidade Católica do Rio Grande do Sul. Porto Alegre, RS, Brasil.

Trabalho elaborado com base nos dados do estudo "Situação de saúde, diagnóstico nutricional e alimentar, imagem corporal, uso de medicamentos e hábitos de vida da população idosa de Campina Grande/PB: uma pesquisa domiciliar".

Apoio financeiro: FAPESQ/CNPQ/UEPB - edital no 002/05.
}

Palavras-chave: Idoso. Grupos Populacionais. Estudo Comparativo. Antropometria. Padrões de Referência. 


\section{Abstract}

This study aimed to describe the anthropometric information on elderly people in Campina Grande, PB, Brazil. It is a populational, cross-sectional household-based study. Data were presented as means, standard deviations and percentiles $\left(5^{\text {th }}, 10^{\text {th }}, 25^{\text {th }}\right.$, $50^{\text {th }}, 75^{\text {th }}, 90^{\text {th }}$ and $\left.95^{\text {th }}\right)$ for weight, stature, body mass index (BMI), mid upper arm circumference (MAC), triceps skinfold thickness (TST), arm muscle circumference (AMC), calf circumference (CC) and waist circumference (WC). The analyses included Student $t$ test to detect differences in mean values of the variable between both sexes. Age impact was investigated by ANOVA. A total of 806 elderly people were interviewed. The mean values of the all variables presented differences statistically significant. The mean values of weight, stature, AMC, CC and WC were higher in men, whereas the mean values of BMI, MAC and TST were higher in women. All variables showed a decrease in average values and percentile $\left(50^{\text {th }}\right)$ with advanced age. The anthropometric and body composition data of the elderly in the present study showed similar trend of others studies, but with different values. Thereby, such values could be useful in the anthropometric assessment of elderly people.
Key words: Elderly. Population Groups. Comparative Study Anthropometry. Reference Standard.

\section{INTRODUÇÃO}

O processo de envelhecimento envolve mudanças corporais, tais como diminuição do peso corporal, da estatura e da massa muscular, bem como redistribuição da gordura corporal, que por sua vez diminui nos membros e aumenta no tronco. ${ }^{1,2}$ A dimensão dessas mudanças pode ter implicações importantes na saúde do idoso. A diminuição da massa muscular contribui para o desenvolvimento de limitações funcionais e incapacidades, afetando diretamente a vida do idoso. ${ }^{3,4} \mathrm{O}$ índice de massa corporal (IMC) tem sido reconhecido como importante preditor de mortalidade e morbidade. Em indivíduos que apresentem IMC elevado, as causas de morte mais associadas são diabetes mellitus, doenças cerebrovasculares, cardiovasculares e infarto. Em relação ao IMC baixo, no entanto, o mesmo constitui fator de risco importante para tuberculose, doença pulmonar obstrutiva e determinados tipos de câncer, como pulmão e estômago. ${ }^{5}$ Em grupos etários mais avançados, o IMC tende a diminuir, sobretudo pela diminuição do peso, ocasionada pela redução da água corporal, perda de massa óssea e muscular. ${ }^{5} \mathrm{O}$ aumento do tecido adiposo na região abdominal é considerado fator de risco importante para o desenvolvimento de doenças cardíacas, hipertensão arterial, dislipidemias e diabetes mellitus. ${ }^{6,7}$ Com isso, torna-se importante a avaliação antropométrica e da composição corporal do idoso, de forma a verificar seu estado nutricional, bem como o risco para o desenvolvimento de determinadas doenças. ${ }^{8,9}$

A antropometria tem se mostrado como importante instrumento na avaliação nutricional do idoso, tanto na prática clínica como em pesquisas epidemiológicas, uma vez que proporciona informações de diferentes componentes da estrutura corporal, principalmente gordura e massa muscular. ${ }^{10}$

As mudanças corporais decorrentes do processo de envelhecimento acontecem de forma diferenciada entre homens e mulheres e nas diferentes fases do envelhecimento; consequentemente, não é aconselhável que padrões antropométricos derivados de uma população adulta sejam utilizados para a avaliação da população idosa. ${ }^{11}$ Estudos com idosos descrevendo algumas informações acerca de suas características antropométricas vêm sendo realizados há algum tempo, principalmente em países desenvolvidos. ${ }^{12-15}$ Apenas recentemente no Brasil, alguns estudos têm sido desenvolvidos com o intuito de fornecer informações antropométricas de referência para a população idosa, seja em estudos populacionais ${ }^{16,17}$ ou com idosos institucionalizados..$^{18}$ No Nordeste, Menezes \& Marucci $^{17}$, em estudo populacional 
com idosos de Fortaleza, sugerem que as informações obtidas com o estudo sejam utilizadas como padrão de referência para os idosos da cidade. No entanto, não foi encontrado na literatura estudo populacional realizado na Paraíba, por conseguinte, em Campina Grande.

O crescimento da população idosa na Paraíba vem acompanhando a tendência brasileira e mundial, representando, de acordo com o Censo 2010, 11,4\% da população paraibana..$^{19}$ Esse aumento da população idosa demanda a condução de estudos que avaliem as condições de saúde dessa população.

Este estudo constitui o primeiro estudo domiciliar, de base populacional, realizado com os idosos de Campina Grande, com o objetivo de caracterizar o perfil antropométrico dessa população. As informações deste estudo poderão ser usadas para comparação das variáveis antropométricas, bem como para a avaliação do estado nutricional dos idosos.

\section{MATERIAL E MÉTODOS}

Este trabalho é parte de um estudo mais amplo que constitui o primeiro inquérito domiciliar com enfoque multidimensional da saúde dos idosos de Campina Grande - PB, e se caracteriza por ser populacional, de base domiciliar, do tipo transversal, com coleta de dados primários. A população-alvo deste estudo foram indivíduos com 60 anos ou mais, de ambos os sexos e residentes habituais em domicílios particulares da zona urbana da cidade de Campina Grande PB. Foram excluídos os idosos em fase terminal de doença, sem possibilidades terapêuticas e que apresentassem debilidade clínica grave; e os que estavam ausentes do município durante o tempo da pesquisa de campo naquele setor censitário.

A amostra foi calculada estimando-se uma prevalência dos desfechos de, no mínimo, $50 \%$. O cálculo do tamanho amostral foi realizado com base na seguinte equação: $\left\{\left[E^{2} \times \mathrm{p}(1-\mathrm{p})\right] \times \mathrm{c}\right\} / \mathrm{A}^{2}$. Onde $E$ é o limite de confiança $(1,96), c$ é o coeficiente de correção amostral $(2,1)$, tendo em vista que a amostra é por conglomerado, e A é a precisão aceita para a prevalência estimada $(A=5 \%)$. Com isso, a amostra foi estimada em 806 idosos.

Para a seleção da amostra, foi utilizada a metodologia adotada pelo Instituto Brasileiro de Geografia e Estatística (IBGE), que divide as cidades em setores censitários, os quais foram obtidos de forma aleatória dentro de cada distrito, bem como os idosos a serem estudados. A amostra deste estudo é constituída por idosos residentes em 24 setores censitários selecionados para comporem a amostra do estudo base.

A Secretaria Municipal de Saúde utiliza, em seus trabalhos, uma organização territorial que divide Campina Grande em seis Distritos Sanitários; sendo assim, foram sorteados quatro setores censitários em cada distrito sanitário. Idosos residentes em unidades constituídas por estabelecimentos coletivos como hospitais, clínicas, escolas e igrejas, clubes, quartéis e similares, mesmo que destinados aos idosos, foram excluídos da seleção aleatória, por conseguinte, do estudo.

A obtenção da amostra ocorreu da seguinte forma: todos os quarteirões de cada setor censitário foram numerados, para posterior sorteio. Com a identificação do quarteirão, o próximo passo foi a escolha do domicílio que ocorreu considerando como ponto de início a esquina inicial do quarteirão, em sentido horário. A cada domicílio selecionado, foram saltados um número de nove domicílios, de acordo com cada setor censitário, e assim sucessivamente, a fim de se obter melhor distribuição. Esse número corresponde à razão entre o número total de domicílios do setor e o número de idosos a serem entrevistados no mesmo setor.

Caso não residisse idoso na casa selecionada, ou se o mesmo não aceitasse ou não pudesse participar do estudo, outro idoso seria procurado no domićlíio seguinte, seguindo a sistemática de seleção. No caso de residir mais de um idoso no domicílio, todos os idosos do domicílio foram convidados a participar do estudo. Nos casos em que residia idoso no domicílio, mas o mesmo estava ausente, foram realizadas até duas visitas de retorno. 
O trabalho de campo, realizado de abril a setembro de 2007, foi desenvolvido por três duplas de entrevistadores, alunos, bolsistas e voluntários, de cursos de graduação da área da saúde, os quais foram devidamente treinados. Durante o treinamento, para garantir a confiabilidade das aferições, as diferenças máximas admitidas nos valores de medidas intra e inter avaliador foram padronizadas de acordo com o proposto por Lohman et al. ${ }^{20}$ Os valores das aferições realizadas pelos entrevistadores foram comparados àqueles obtidos pela professora coordenadora da pesquisa, a qual possui experiência em outras pesquisas antropométricas. ${ }^{17,18}$

As variáveis antropométricas avaliadas foram: peso ( $\mathrm{kg}$ ), estatura (m), índice de massa corporal (IMC) $\left(\mathrm{kg} / \mathrm{m}^{2}\right)$, perímetro do braço (PB) $(\mathrm{cm})$, dobra cutânea tricipital (DCT) (mm), circunferência muscular do braço (CMB) $(\mathrm{cm})$, perímetro da panturrilha $(\mathrm{PP})(\mathrm{cm})$ e perímetro da cintura (PC) (cm), as quais foram aferidas com base nas técnicas propostas por Lohman et al. ${ }^{20}$

Para o cálculo do índice de massa corporal (IMC), que consiste na medida do peso corporal $(\mathrm{kg})$, dividido pela estatura (m) elevada ao quadrado $\left(\mathrm{P} / \mathrm{E}^{2}\right)$, foram mensurados a estatura e o peso. Para a mensuração da estatura foi utilizado o antropômetro portátil, com escala de $220 \mathrm{~cm}$ e precisão de $1 \mathrm{~mm}$ (Altura Exata). Para mensuração do peso, foi utilizada balança eletrônica digital portátil, tipo plataforma, com capacidade para $150 \mathrm{~kg}$ e sensibilidade de $100 \mathrm{~g}$ (TANITA UM080).

A dobra cutânea tricipital foi aferida utilizando o compasso Lange, que tem pressão constante de $10 \mathrm{~g} / \mathrm{mm}^{2}$. O perímetro do braço (PB), o perímetro da panturrilha (PP) e o perímetro da cintura $(\mathrm{PC})(\mathrm{cm})$ foram mensurados utilizando fita métrica inelástica, com precisão de $1 \mathrm{~mm}$.

A partir dos valores de DCT e $\mathrm{PB}$, foi calculada a circunferência muscular do braço (CMB), utilizando a seguinte equação: ${ }^{21} \mathrm{CMB}$ $(\mathrm{cm})=\mathrm{PB}(\mathrm{cm})-(\pi \times \mathrm{DCT}(\mathrm{mm}))$. As variáveis antropométricas são apresentadas sob a forma de média, desvio-padrão (DP) e percentil (P5, P10, P25, P50, P75, P90, P95).
O banco de dados foi elaborado utilizandose o aplicativo Microsoft Office Excel 7.0. As informações estatísticas foram obtidas com o auxílio do aplicativo estatístico Statistical Package for the Social Science (SPSS 17.0). Em todas as análises foi adotado um intervalo de confiança de $95 \%$. A significância estatística das diferenças de médias entre os sexos foi verificada por meio do teste $t$ Student. Foram realizadas a análise de variância (ANOVA) e a comparação de Tukey's para verificar o efeito do grupo etário (60 a 69 anos, 70 a 79 anos, 80 anos ou mais) nas variáveis antropométricas. Foi utilizado o nível de significância $\alpha=5 \%$.

Todos os idosos assinaram o Termo de Consentimento Livre e Esclarecido depois de receberem explicações verbais e escritas a respeito do estudo. O estudo maior, do qual este faz parte, foi aprovado pelo Comitê de Ética em Pesquisa da Universidade Estadual da Paraíba (UEPB), sob o protocolo no. 0136.0.133.000-06, não havendo conflito de interesses.

\section{RESULTADOS}

O número total de idosos entrevistados no estudo maior foi 806 (69,1\% mulheres); no entanto, o número de idosos foi diferente para cada variável antropométrica, tendo em vista que em alguns idosos não foi possível aferi-las, seja por recusa ou por impossibilidade dos mesmos. A média etária dos idosos foi 72,4 anos (DP = 8,7 anos).

$\mathrm{Na}$ tabela 1 são apresentadas informações das características antropométricas, de acordo com o sexo e grupo etário. Os valores médios das variáveis peso, estatura, CMB, PP e PC foram significativamente maiores entre os homens que entre as mulheres, enquanto que os valores médios de IMC, PB e DCT foram significativamente maiores entre as mulheres, quando comparadas aos homens.

Nessa tabela é possível observar, ainda, os valores médios das variáveis de acordo com o grupo etário. Todas as variáveis apresentaram valores médios menores nos grupos etários mais 
avançados, principalmente quando se observa os valores dos 60 a 69 anos e dos 80 ou mais. No entanto, em algumas variáveis estas diferenças não foram estatisticamente significativas, como é o caso do IMC, DCT e PC, entre os homens, e PC entre as mulheres. A diferença dos valores médios se mostrou estatisticamente significativa, tanto entre os homens como entre as mulheres, para as variáveis peso, estatura, $\mathrm{PB}, \mathrm{CMB}$ e $\mathrm{PP}$, assim como para IMC e DCT entre as mulheres.

$\mathrm{Na}$ tabela 2, são apresentados os percentis das variáveis antropométricas, onde é possível observar, de forma mais detalhada, essa tendência de declínio de acordo com o grupo etário.

\section{DISCUSSÃO}

O presente estudo descreve o perfil antropométrico de uma amostra representativa da população idosa de Campina Grande, Paraíba, Brasil. Todos os idosos participantes da pesquisa maior, da qual este estudo faz parte, foram convidados a participarem da avaliação antropométrica. No entanto, devido a recusa ou limitações individuais para aferição de determinadas medidas (acamados, cadeirantes, presença de edema no braço ou perna), não foi realizada a aferição de todas as variáveis em todos os idosos.

O processo de envelhecimento acarreta alterações físicas que podem influenciar nas dimensões corporais. Em geral, o peso tende a elevar na fase adulta e diminuir progressivamente com a idade, numa proporção de aproximadamente $1 \mathrm{~kg}$ por década. A estatura também tende a diminuir, podendo chegar a uma diminuição de 0,5 a $1,5 \mathrm{~cm}$ por década. ${ }^{22,23}$ Estudos têm mostrado que o peso e a estatura tendem a ser maiores entre os homens, quando comparados às mulheres, e que ambos diminuem gradualmente com o avançar da idade, tanto em homens como em mulheres. ${ }^{1,14,16,18,24-26}$

Corroborando os resultados de outras pesquisas, o peso e a estatura dos idosos deste estudo foram maiores entre os homens, assim como ambas as variáveis diminuíram com o avançar do grupo etário. Essa diminuição da estatura se deve, principalmente, a compressão vertebral, perda de tônus muscular e problemas posturais. ${ }^{5}$

Coqueiro et al., ${ }^{26} \mathrm{em}$ estudo populacional com idosos em Havana, Cuba, verificaram média de peso e estatura dos homens, superior à das mulheres.Sánchez-Garcíaetal., estudandoidosos no México, verificaram diferenças significativas entre os valores médios de peso e estatura entre homens e mulheres. Os autores observaram que as médias de peso e estatura das mulheres foram $62,7 \pm 11,6 \mathrm{~kg}$ e $152,6 \pm 7,5 \mathrm{~cm}$, respectivamente, enquanto que dos homens foram $70,3 \pm 12,3 \mathrm{~kg}$ e $163,2 \pm 8,5 \mathrm{~cm}$, respectivamente. Esses valores foram superiores aos encontrados no presente estudo. Diferenças nos valores médios das variáveis antropométricas, entre regiões e países, têm sido observadas em diferentes estudos. ${ }^{11,12}$

Semelhantemente a outras pesquisas, ${ }^{1,14,25,26}$ os valores médios do IMC dos idosos deste estudo foram significativamente superiores entre as mulheres quando comparadas aos homens. Arroyo et al., ${ }^{27}$ em estudo com idosos no Chile, encontraram média do IMC das mulheres $(28,5$ $\left.\mathrm{kg} / \mathrm{m}^{2}\right)$ superior à dos homens $\left(27,4 \mathrm{~kg} / \mathrm{m}^{2}\right)$, cuja diferença se mostrou estatisticamente significativa. De acordo com a World Health Organization, ${ }^{5}$ o ganho de peso nos homens comumente atinge o platô por volta dos 65 anos e geralmente declina. Nas mulheres esse platô ocorre por volta dos 75 anos, de modo que, enquanto os homens param de ganhar peso, as mulheres continuam ganhando, por conseguinte, elevando o IMC.

Diferenças nas médias de IMC dos idosos, com o avançar do grupo etário, foram observadas tanto em homens como em mulheres, mostrando um possível efeito da idade na diminuição dessa variável. Essa tendência de diminuição do IMC com o avançar da idade vem sendo observada por outros pesquisadores em estudos transversais e longitudinais. ${ }^{1,13,16,24,26,28}$ Essa diminuição do IMC com a idade pode ser devida à redução da massa corporal magra e à diminuição da quantidade de gordura e água no organismo. ${ }^{5}$

Com relação aos dados de IMC, em percentil, observa-se que $50 \%$ das mulheres apresentam 
IMC abaixo de $26,8 \mathrm{~kg} / \mathrm{m}^{2}$ e que $50 \%$ dos homens apresentam IMC abaixo de 25,1 kg/ $\mathrm{m}^{2}$. Esse fato é preocupante, tendo em vista que em pesquisa realizada por Kvamme et al., ${ }^{29}$ para avaliar o impacto do IMC na mortalidade de idosos, verificou-se que IMC abaixo de $25 \mathrm{~kg} / \mathrm{m}^{2}$, tanto entre os homens como entre as mulheres, esteve associado com elevada mortalidade. Menezes \& Marucci, ${ }^{18}$ em pesquisa antropométrica com idosos institucionalizados de Fortaleza, verificaram que $75 \%$ das idosas estudadas apresentavam valores de IMC abaixo de $26,9 \mathrm{~kg} / \mathrm{m}^{2}$ e que $75 \%$ dos idosos apresentavam valores de IMC abaixo de $25,5 \mathrm{~kg} / \mathrm{m}^{2}$. Essa diferença entre os estudos pode se dever ao fato de os idosos avaliados por Menezes \& Marucci ${ }^{18}$ serem institucionalizados, tendo em vista que estudos com idosos institucionalizados têm observado menores valores médios de IMC nesse grupo. ${ }^{30,31}$

Os valores médios do perímetro do braço $(\mathrm{PB})$ dos idosos deste estudo foram similares entre homens e mulheres, com os homens apresentando valores maiores. Em estudo realizado por Arroyo et al., ${ }^{27}$ observou-se que homens e mulheres apresentaram pequena diferença entre os valores do $\mathrm{PB}$, a qual não apresentou diferença estatisticamente significativa. Com relação ao grupo etário, tanto homens como mulheres apresentaram diminuição dos valores médios com o avançar da idade. Assim como neste estudo, outros autores têm observado valores similares entre os sexos e diminuição dos valores ao longo dos anos. ${ }^{1,13,17,18,24,26}$

O perímetro do braço constitui a soma dos tecidos ósseo, muscular, gorduroso e epitelial do braço. Dessa forma, a diminuição do PB, observada nos idosos deste estudo, tanto nos valores médios como em percentil, pode ser devida à diminuição da massa muscular e do tecido adiposo dos membros, naturais do processo de envelhecimento. ${ }^{1,2}$

A dobra cutânea tricipital (DCT) é uma variável antropométrica utilizada como indicadora da reserva de gordura subcutânea e comumente utilizada em estudos com idosos. ${ }^{16-18,24,26,27}$ Ovalormédio da DCT dasidosas deste estudo foi significativamente superior $(22,0 \mathrm{~mm})$ ao dos homens $(13,4 \mathrm{~mm})$, sugerindo que as mulheres apresentam maior reserva de gordura. Menezes \& Marucci ${ }^{17}$ encontraram valores semelhantes aos encontrados neste estudo, onde a média de DCT das mulheres $(21,3 \mathrm{~mm})$ foi significativamente superior à dos homens $(13,0 \mathrm{~mm})$. Achados semelhantes foram observados em outros estudos, nos quais mulheres apresentam maior reserva de gordura que os homens. ${ }^{16-18}$

Tanto os homens como as mulheres deste estudo apresentaram diminuição dos valores médios ao longo dos grupos etários. Essa diminuição, no entanto, foi mais pronunciada entre as mulheres, cuja diferença entre as médias foi significativa. Entre os homens, houve discreta diminuição. Essa tendência de diminuição dos valores médios de DCT com o avançar da idade foi semelhante à encontrada em outros estudos. ${ }^{13,16-18,26}$

A circunferência muscular do braço (CMB) tem se mostrado um bom indicativo de reserva de massa muscular e, por isso, utilizada em estudos antropométricos. ${ }^{13,16-18,26}$ A CMB dos idosos deste estudo apresentou valores médios maiores entre os homens, quando comparados às mulheres, cujas diferenças se mostraram estatisticamente significativas. Além disso, os valores médios desta variável declinaram significativamente com o avançar do grupo etário em ambos os sexos. De acordo com os dados do estudo de Menezes \& Marucci, ${ }^{18}$ os valores médios da CMB apresentaram decréscimo com o avançar da idade e associação significativa com o sexo, sendo mais acentuado no sexo feminino. Nos homens, não houve diferença significativa entre os grupos etários. Um fato peculiar, exposto pelos pesquisadores, se refere ao aumento ínfimo e sem significância da CMB em idosos com 80 anos ou mais.

Estudos antropométricos com idosos têm utilizado, além da CMB, outras variáveis indicadoras de reserva de massa muscular como área muscular do braço (AMB) e área muscular do braço corrigida (AMBc), os quais, independentemente do grupo de idosos 
estudado, têm observado um padrão similar ao encontrado neste estudo, no qual os homens apresentam maior depósito de massa muscular, assim como sua diminuição com o avançar do grupo etário, que ocorre tanto em homens como em mulheres, mas de forma mais pronunciada entre os homens. ${ }^{13,15-17,26}$

A diminuição nos valores das variáveis indicativas de reserva de massa muscular, de acordo com o grupo etário, é preocupante, uma vez que alterações musculares levam a manifestações clínicas que podem influenciar de forma negativa na vida do idoso, visto que essa perda tem impacto sobre sua capacidade funcional. ${ }^{22}$ Estudo realizado por Rebelatto et al. ${ }^{32}$ detectou que idosos que sofriam quedas apresentavam força e reserva de massa muscular significativamente inferiores aos que não caíam. Dessa forma, o grau do decréscimo de massa muscular parece ser um determinante importante para a ocorrência de quedas.

Além das variáveis indicativas de reserva de massa muscular citadas anteriormente, o perímetro da panturrilha (PP) também tem sido utilizado para este fim, sendo considerado um bom indicador de massa muscular em idosos. ${ }^{5}$ Vellas, Garry \& Guigoz, ${ }^{33}$ desenvolvendo um instrumento para identificar idosos em risco de má nutrição (Mini Nutritional Assessment - MNA), apontam como aconselhável que os valores do PP sejam iguais ou superiores a $31 \mathrm{~cm}$. Ao considerar esse valor, observa-se que os valores médios do PP dos idosos deste estudo foram superiores $(34,5 \mathrm{~cm}$ nas mulheres e $34,8 \mathrm{~cm}$ nos homens). De acordo com os dados em percentil, observa-se que aproximadamente $10 \%$ dos idosos do sexo feminino apresentaram valores menores que $30,7 \mathrm{~cm}$ e $25 \%$ das mulheres valores menores que $31,8 \mathrm{~cm}$.

Os estudos têm comumente verificado idosos com valores médios de PP superiores a 31,0 cm. ${ }^{1,24,27,34}$ Apesar de o valor sugerido por Vellas, Garry \& Guigoz $^{33}$ não diferenciar a avaliação dos idosos por sexo, os estudos têm mostrado valores de PP superiores entre os homens. Além disso, os valores tendem a diminuir com a idade, tanto em homens como em mulheres.,15,24,27
Estudo realizado por Portero-McLellan et al., ${ }^{34}$ objetivando verificar se o PP poderia ser utilizado para monitorar o estado nutricional de idosos hospitalizados, encontrou correlação positiva entre o PP e o estado nutricional dos idosos, sugerindo que a variável pode ser utilizada como medida complementar no monitoramento nutricional destes idosos.

O perímetro da cintura (PC) é uma variável que fornece uma estimativa da gordura abdominal e constitui um dos mais acessíveis métodos de verificação da gordura visceral. ${ }^{35}$ Quanto a essa variável antropométrica, pode-se perceber, neste estudo, que houve diminuição dos valores médios com o avançar do grupo etário entre as mulheres, em que o grupo de 6069 anos apresentou valores maiores que os demais grupos. Entre os homens, o grupo com 70-79 anos apresentou valores médios discretamente maiores que o grupo de 60-69 anos. Em ambos os sexos, o grupo de 80 anos ou mais apresentou os menores valores.

De acordo com a II Diretriz Brasileira de Cardiogeriatria, ${ }^{35}$ são considerados adequados valores de PC inferiores a $88 \mathrm{~cm}$ para as mulheres e inferiores a $102 \mathrm{~cm}$ para homens. Neste estudo, o valor médio do PC das mulheres foi discretamente inferior a esses valores. $\mathrm{O}$ valor médio dos homens foi inferior, ou seja, compatível com o preconizado pela II Diretriz. ${ }^{35}$ A apresentação dos dados em percentil mostra que apenas $50 \%$ das mulheres apresentaram valores de PC inferiores a $87,5 \mathrm{~cm}$ e que $90 \%$ dos homens apresentaram valores inferiores a 103,4 cm. Estudos com idosos comumente verificam elevados valores de perímetro da cintura nesta população, principalmente entre as mulheres. ${ }^{14,16,26,27}$ Fato preocupante, visto que a obesidade abdominal é atualmente um sério agravo à saúde que, associada a doenças cardiovasculares, hipertensão e resistência insulínica, caracterizam fatores de risco para a síndrome metabólica. ${ }^{35}$

Este trabalho, assim como outros estudos transversais, apresenta limitações características desse tipo de estudo, tendo em vista que o mesmo não permite avaliar a magnitude das 
alterações nas variáveis antropométricas com o envelhecimento. Com isso, observa-se a necessidade de estudos longitudinais acerca da ocorrência dessas alterações e os prováveis fatores associados.

\section{CONCLUSÃO}

Os resultados verificados neste estudo confirmam estudos anteriores, transversais e longitudinais, os quais têm verificado resultados semelhantes quanto à influência da idade nas características antropométricas e de composição corporal dos idosos.

\section{REFERÊNCIAS}

1. Sánchez-García S, García-Peña C, Duque-López MX, Juárez-Cedillo T, Cortés-Núñez AR, ReyesLópes S. Anthropometric measures and nutritional status in a healthy elderly population. BMC Public Health 2007;7:2-10.

2. Atlantis E, Martin SA, Haren MT, Taylor AW, Wittert GA. Lifestyle factors associated with age-related differences in body composition: the Florey Adelaide Male Aging Study. Am J Clin Nutr 2008;88:95-104.

3. Morley JE, Baumgartner RN, Roubenoff R, Mayer J, Nair KS. Sarcopenia. J Lab Clin Med 2001;137:231-43.

4. Janssen I. Influence of sarcopenia on the development of physical disability: the Cardiovascular Health Study. J Am Geriatr Soc 2006;54:56-62.

5. World Health Organization. Physical status: the use and interpretation of anthropometry. Technical Report Series 1995;854:375-409.

6. Snijder MB, Zimmet PZ, Visser M, Dekker JM, Shaw JE. Independent and opposite associations of waist and hip circumferences with diabetes, hypertension and dyslipidemia: the AusDiab Study. Int J Obes Relat Metab Disord 2004;28(3):402-09.

7. Huxley R, Mendis S, Zheleznyakov E, Reddy S, Chan J. Body mass index, waist circumference and waist:hip ratio as predictors of cardiovascular risk - a review of the literature. Eur J Clin Nutr 2010;64(1):16-22.

8. Forster S, Gariballa S. Age as a determinant of nutritional status: a cross sectional study. Nutr J 2005;4:28-32.
Apesar dessa similaridade na tendência com outros estudos, é importante ressaltar a presença de diferenças entre os valores médios e percentis, o que possivelmente acontece por conta das diferenças entre raça, localidade, região e país. Sendo assim, torna-se cada vez mais importante o conhecimento específico das características antropométricas de cada grupo etário, possibilitando a construção de padrões locais de referência. Diante disso, sugere-se que as informações deste estudo possam ser utilizadas na avaliação de idosos em Campina Grande e demais áreas urbanas do Nordeste, tendo em vista a existência de poucos estudos desse tipo na região.

9. Villareal DT, Apovian CM, Kushner RF, Klein $S$; American Society for Nutrition; NAASO. The Obesity Society: Obesity in older adults: technical review and position statement of the American Society for Nutrition and NAASO, The Obesity Society. Am J Clin Nutr 2005;82:923-34.

10. Baumgartner RN. Body composition in elderly persons: a critical review of needs and methods. Prog Food Nutr Sci 1995;17:223-60.

11. Rosnah MY, Mohd Rizal H, Sharifah-Norazizan SAR. Anthropometry dimensions of older Malaysians: comparison of age, gender and ethnicity. Asian Social Science 2009;5(6):133:140.

12. Delarue J, Constans T, Malvy D, Pradignac A, Couet C, Lamisse F. Anthropometric values in an elderly French population. Brit J Nutr 1994;71:295-302.

13. Kuczmarski MF, Kuczmarski RJ, Najjar M. Descriptive anthropometric reference data for older Americans. J Am Diet Assoc 2000;100:59-66.

14. Perissinotto E, Pisent C, Sergi G, Grigoletto F, Enzi G; ILSA Working Group (Italian Longitudinal Study on Ageing). Anthropometric measurements in the elderly: age and gender differences. Brit J Nutr 2002;87:177-86.

15. Corish CA, Kennedy NP. Anthropometric measurements from a cross-sectional survey of Irish free-living elderly subjects with smoothed centile curves. Brit J Nutr 2003;89:137-45. 
16. Barbosa RB, Souza JM, Lebrão ML, Marucci Mde FN. Anthropometry of elderly residents in the city of São Paulo, Brazil. Cad Saúde Pública 2005;21 (6):1929-38.

17. Menezes TN, Marucci MF. Perfil dos indicadores de gordura e massa muscular corporal dos idosos de Fortaleza, Ceará, Brasil. Cad Saúde Pública 2007;23(12):2887-95.

18. Menezes TN, Marucci MFN. Antropometria de idosos residentes em instituições geriátricas, Fortaleza, CE. Rev Saúde Pública 2005;39(2):169-75.

19. Instituto Brasileiro de Geografia e Estatística. Dados Censo 2010 publicados no Diário Oficial da União. 04 nov 2010.

20. Lohman TG, Roche AF, Martorell R. Anthropometric standardization reference manual. Champaign, Illinois: Human Kinetics Books, 1988.

21. Gurney JM, Jelliffe DB. Arm anthropometry in nutritional assessment: nomogram for rapid calculation of muscle circumference and crosssectional muscle and fat areas. Am J Clin Nutr 1973;26(9):912-5.

22. Chumlea WC, Baumgartner RN. Status of anthropometric and body composition data in elderly subjects. Am J Clin Nutr 1989;50(5 Suppl):1158-66.

23. Baumgartner R, Chumlea WC, Roche AF. Estimation of body composition from bioelectric impedance of body segments. Am J Clin Nutr 1989;50(2):221-26.

24. Santos JL, Albala C, Lera L, García C, Arroyo P, Pérez-Bravo F, et al. Anthropometric measurements in the elderly population of Santiago, Chile. Nutrition 2004;20(5):452-7.

25. Zverev Y, Chisi J. Anthropometric indices in rural Malawians aged 45-75 years. Ann Human Biol 2004;31(1):29-37.

26. Coqueiro Rda S, Barbosa AR, Borgatto AF. Anthropometric measurements in the elderly of Havana, Cuba: age and sex differences. Nutrition 2009;25(1):33-9.
27. Arroyo P, Lera L, Sánchez H, Bunout D, Santos JL, Albala C. Indicadores antropométricos, composición corporal y limitaciones funcionales em ancianos. Rev Méd Chile 2007;135:846-54.

28. Dey DK, Rothenberg E, Sundh V, Bosaeus I, Steen B. Height and body weight in the elderly. I. A 25-year longitudinal study of a population aged 70 to 95 years. Eur J Clin Nutr 1999;53(12):905-14.

29. Kvamme JM, Holmen J, Wilsgaard T, Florholmen J, Midthjell K, Jacobsen BK. Body mass index and mortality in elderly men and women: the Tromso and HUNT studies. J Epidemiol Communit Health 2012;66(7):611-7.

30. Mowe M, Bohmer T. Nutrition problems among home-living elderly people may lead to disease and hospitalization. Nutr Rev 1996;54(1 Pt 2):22-4.

31. Rauen MS, Moreira EAM, Calvo MCM, Lobo AS. Avaliação do estado nutricional de idosos institucionalizados. Rev Nutr 2008;21(3):303-10.

32. Rebelatto JR, Castro AP, Chan A. Quedas em idosos institucionalizados: características gerais, fatores determinantes e relações com a força de preensão manual. Acta Ortop Bras 2007;15(3):151-4.

33. Vellas BJ, Garry PJ, Guigoz Y; Nestlé Nutrition Services. Mini nutritional assessment (MNA): research and practice in the elderly. Nestlé Nutrition Workshops Series Clinical \& Performance Programme v.1. Karger Publishers. 1999.

34. Portero-McLellan KC, Staudt C, Silva FR, Delbue Bernardi JL, Baston Frenhani P, Leandro Mehri VA. The use of calf circumference measurement as an anthropometric tool to monitor nutritional status in elderly inpatients. J Nutr Health Aging 2010;14(4):266-70.

35. Gravina CF, Rosa RF, Franken RA, Freitas EV, Liberman A, et al; Sociedade Brasileira de Cardiologia. II Diretriz Brasileira em Cardiogeriatria. Arq Bras Cardiol 2010;95(3 Supl 2):1-112. 